\title{
CLINICAL OUTCOME OF INTERSPHINCTERIC RESECTION FOR ULTRA-LOW RECTAL CANCER
}

\author{
Valentin L. Ignatov, Nikola Y. Kolev, Anton Y. Tonev, Georgi H. Ivanov, Aleksander \\ K. Zlatarov, Georgi Todorov, Velian Platikanov, Krasimir D. Ivanov \\ 1st Clinic of Surgery, University Hospital "St. Marina", Varna, Bulgaria
}

\begin{abstract}
BACKGROUND: Laparoscopic surgery has been reported to be one of the approaches for total mesorectal excision (TME) in rectal cancer surgery. Intersphincteric resection (ISR) has been reported as a promising method for sphincter-preserving operation in selected patients with very low rectal cancer. METHODS: We try to underline the important surgical issues surrounding the management of patients with low rectal cancer indicated to laparoscopic intersphincteric resection (ISR). From January 2007 till now, 35 patients with very low rectal cancer underwent laparoscopic TME with ISR. We report and analyze the results from them

RESULTS: Conversion to open surgery was necessary in one $(3 \%)$ patient. The median operation time was $293 \mathrm{~min}$ and median estimated blood loss was $40 \mathrm{ml}$. The pelvic plexus was completely preserved in 32 patients. There was no mortality. Postoperative complications occurred in three $(9 \%)$ patients. The median length of postoperative hospital stay was 11 days. Macroscopic complete mesorectal excision was achieved in all cases. Complete resection (R0) was achieved in 21 (91\%) patients.

CONCLUSIONS: Laparoscopic TME with ISR is technically feasible and a safe alternative to laparotomy with favorable short-term postoperative outcomes. The literature research made by us found that the laparoscopic approach can be underwent in most patients with low rectal cancer in which laparoscopic ISR represents a feasible alternative to conventional open surgery.
\end{abstract} cancer.

Key words:intersphincteric resection, low rectal

\section{INTRODUCTION}

In earlier ages, tumors within $7-8 \mathrm{~cm}$ above the anal verge were treated by abdominoperineal resection (APR), especially when the rectal tumor could be easily palpated by an examiner's finger. Along with the recent development of circular stapling devices, it facilitates the feasibility of ultra-low anterior resection of rectal tumor. Recent studies have shown that a distal clearance margin greater than 1.5 $\mathrm{cm}$ is sufficient when tumor histological differentiation is not poor to achieve curative resection of low rectal tumors[1]. These have established the feasibility of ultralow anterior resection for tumors at level as low as $3 \mathrm{~cm}$ from the dentate line.

For the ultra-low tumors, i.e., tumor with lower margin situated within $1-3 \mathrm{~cm}$ above the dentate line, the mandatory surgical procedure is still controversial. It is hardly practical to apply conventional ultra-low anterior resection with autosuture instrumentation to achieve an adequate section margin, because of the extreme difficultly in placing a stapler across the optimal distal rectal margin. While most surgeons insist in performing APR in these cases, intersphincteric resection (ISR) has drawn increasing attention as it provides anus preservation and more clear vision for resection.

Over the past 10 years, laparoscopy has become the gold standard for the surgical management of many digestive diseases, including benign colorectal pathologies. However, the oncologic safety of this approach is still controversial, so laparoscopic methods have been less widely applied to colorectal malignancy. Excellence of surgical technique is of particular relevance in the treatment of rectal cancer. Routine excision of the intact mesorectum during resection of cancers of the middle and lower rectum has resulted in the lowest incidence of local recurrences ever reported. These standards, established by Heald et al., are those against which any new technique must be evaluated. Many surgeons have therefore argued that given the current status of laparoscopic technology, the steep learning curve of colorectal laparoscopic procedures, and their uncertain oncologic efficacy, carcinoma of the rectum should be excised only by specialized colorectal surgeons using conventional techniques. 2

At our institutions we started laparoscopic colorectal surgery at the beginning of 2005, considering tumors of the middle and lower rectum as a contraindication to this approach because of presumed technical difficulties in obtaining a radical dissection of the mesorectum. Having obtained a good experience with more than 50 laparoscopic colon resections with satisfactory clinical results and having successfully performed a few laparoscopic abdomino-perineal resections with an excellent view during the pelvic dissection, in 2007 we decided to extend the routine use of the laparoscopic approach to tumors of the 
middle and lower retum.

Many retrospective studies have pointed out that there is a good local control after sphincter-saving resection for rectal cancer[2-11]; however, the majority of studies did not carry more than the 5-year follow-up interval and they examined all rectal tumors, many of which can be removed by low/ultra-low anterior resection. This study assessed the oncological outcome of patients with very low rectal cancer by intersphincteric resection to determine whether abdominoperineal resection could be abolished.

\section{MATERIALS AND METHODS}

607 nonrandomized consecutive patients underwent laparoscopic colorectal resections between June 1994 and June 2001. In this period, of 154 neoplasms located in the low and middle rectum, $100(65 \%)$ were treated by laparoscopic total mesorectal excision (TME). We used followed localization margins $-7 \mathrm{~cm}$ or less from the anal verge, low rectum; 7 to $12 \mathrm{~cm}$, middle rectum. All patients treated in this period were included in a prospective study. The protocol was approved by the ethical committee of each collaborating institution, and patients gave informed consent. In the absence of specifics contraindications to laparoscopy (e.g., severe cardiopulmonary disease, glaucoma), patients with tumors located in the low and middle rectum were selected for laparoscopic TME based on the following criteria: elective surgery, absence of occlusion, American Society of Anesthesiologists (ASA) status I to III. Neither morbid obesity nor prior pelvic surgery was considered a contraindication to laparoscopic TME. When the neoplasm at digital examination reached the anatomic anal canal or was fixed to the pelvic floor, the patient was excluded from the study and a laparoscopic abdominoperineal resection was performed. Therefore, all patients included in this study had a sphincter-saving procedure. Preoperative work-up consisted of clinical evaluation, total colonoscopy, computed tomographic scan, upper abdominal ultrasound, and endoscopic ultrasound. Patients preoperatively staged $\mathrm{T} 3$ or $\mathrm{T} 4$ without distant metastases were treated by preoperative radiochemotherapy (45 Gy during 4 weeks together with systemic 5-buouracil intravenous infusion) and were reevaluated with clinical examination and computed tomographic scan 20 days after the completion of the treatment. Desnitive inclusion in this study was decided at this point, excluding locally advanced tumor (i.e., T4 in the TNM classification. Clinical parameters analyzed included patient characteristics, operative variables, pathologic examination, and short-term and longterm outcomes. Analysis of patient characteristics included age and gender. Operative variables examined included length of operative procedure (from skin incision to the application of dressings) and conversion rate. Conversion to laparotomy was defined as unplanned incision or an incision made longer or earlier than planned. Pathologic examination included type (adenoma or adenocarcinoma) and stage of disease (TNM). Parameters recorded included number of lymph nodes harvested and longitudinal and radial margins of excision. Short-term outcomes included postoperative morbidity, 30-day mortality, and quality of life assessment evaluated by analgesic requirement. Long-term outcomes included tumor recurrence, disease-free survival, and overall survival for rectal cancer. Results were compared with literature data. Patients were followed up with physician examination, digital examination, serum carcinoembryonic antigen (CEA) assay, ultrasound of the liver, computed tomographic scan, chest radiograph, and colonoscopy. Every case of suspected local recurrence was histologically confirmed. Data were collected prospectively from the time of diagnosis using a custom-written computerized database.

\section{METHODS}

ISR was performed according to the methods previously described by Schiessel et al[4] This procedure was initiated by placing the patient in the Sim's position for the anal approach, mucosal incision, further exposure of the internal anal sphincter and intersphincteric space. After meticulous hemostasis was reached in the operation field, followed by closure of the rectal stump, and the patient was placed in lithotomy position to facilitate low anterior resection of the rectum with total mesorectal excision by laparotomy. A colonic J-pouch in all the patients as $6-8 \mathrm{~cm}$ in length was constructed from the distal descending colon and/or proximal sigmoid colon with uniform linear staplers. Mobilization of the splenic flexure colon, descending colon, sigmoid colon and ligation of the inferior mesenteric vein at the inferior margin of the pancreas are critical components of this procedure to enable the pouch to be easily drawn toward the anus. The constituted reservoir was then anastomosed to the dentate line with interrupted sutures. Under direct vision, the external anal sphincter was preserved to maintain defecation function.

During period II, ISR was performed on 10 patients with the same tumor location as period I. Among those, six patients with fixed tumors (T3-4) underwent preoperative CCRT ( 5040 cGy in 28 fractions over 6 wk with continuous infusion of 5-FU and LV on the first and last $5 \mathrm{~d}$ during radiotherapy).

All patients were performed temporary diverting colostomy. Follow-up evaluations were performed on an outpatient basis. As to that whether tumor recurrence or distant metastasis existed, it was determined by digital rectal examination, clinical symptoms, measurement of serum tumor marker level and image study facilities.

\section{RESULTS}

Clinical and pathological data

On the other hand, there were 10 patients recruited in the period II since 1999 till 2003, 5 males and 5 females, 
median age was 62 (range: 42-72) years. No statistic difference in age or gender between period I and period II was proved.

Among the period II group, six patients with fixed tumors were undertaken by the protocol of preoperative CCRT (5 040 cGy in 28 fractions over 6 wk concurring with continuous infusion 5-FU and LV on the first and last $5 \mathrm{~d}$ during radiotherapy). In comparing the tumor staging between pre- and post-CCRT, there were seven patients with a significant tumor regression status, including two complete regressions. Pathologic TNM classifi cations for the 10 patients were $2 \mathrm{~T} 0,1 \mathrm{~T} 1 \mathrm{~N}-, 5 \mathrm{~T} 2 \mathrm{~N}-, 1 \mathrm{~T} 2 \mathrm{~N}+$ and $1 \mathrm{~T} 3 \mathrm{~N}+$.

Curative resection of malignancy with microscopically clear oncologic section margin was confirmed by postoperative pathologic diagnosis in all the patients. The median distal resection margin was 1.5 (range: $0.5-2$ ) $\mathrm{cm}$. There was no postoperative mortality. Postoperative morbidity was found as follows: wound infection in 4/18 $(22.2 \%)$, prolonged Foley catheter indwelling in 3/18 (16.7\%), and urinary tract infection in 2/18 (11.1\%).

All patients underwent interval colostomy closure at a median of 7 (range, 3-14) mo, no colostomy closure related complication was proposed. Postoperative local recurrence and distant metastasis

There was no local recurrence or distant metastasis in these 10 patients after a median follow-up of 30 (range: 18-47) mo

\section{Functional outcome}

Eight patients (80\%) experienced six or fewer bowel movements per day, urgency was reported in 5 patients $(50 \%)$, and most of them could tolerate anal continence status well. Only two patients stated that they were incontinent to liquid stools and required pad use at night time

\section{DISCUSSION}

The distal section margin of 1-2 cm would be currently considered sufficient for ultra-low rectal cancer in most instances[1,5,7,8]. Parks et al[12] reported that long-term survival and local recurrence rate after ISR were similar to those after APR. Several specialized studies have investigated ISR for low rectal cancer, and their local recurrence rates ranged from $0 \%$ to $12 \%[2-11]$; however, some studies reported that the patients with the location of rectal cancer at $5 \mathrm{~cm}$ more proximal to anal verge and did not always carried more than a 5-year follow-up interval. In this study, the local recurrence rate was $12.5 \%$ for T2-staged ultra-low rectal tumor after at least a 5-year follow-up. However, the number of patients in this study is small because of the highly selected criteria, size of the tumors smaller than $5 \mathrm{~cm}$ and the lower margin of tumors within 1 to $3 \mathrm{~cm}$ from the dentate line.

The radial involvement of a tumor is another critical predictive factor for local recurrence after rectal cancer resection. In most instances, patients with T3-4 carcinomas of the low-third rectum require APR. Recently, by using a multimodal approach, intersphincteric resection was practical in patients with T3-4 carcinomas of the lower third of the rectum[7,10,11,13-15]. Preoperative CCRT reducing tumor volume, causing tumor down-staging, and further facilitating surgical resection of malignancy have been proposed. Recent studies concerning preoperative chemoradiotherapy have demonstrated that it improves local control and cancerrelated survival. Rullier et al[10] reported only $2 \%(1 / 43)$ local recurrence rate if combining preoperative CCRT and successive ISR for ultra-low rectal cancer were attempted (median follow-up was 30 mo)[10]. Luppi et al[13] reported 94\% local control rate for T3-4 rectal cancer using preoperative chemoradiotherapy[13]. Saito et al reported similar results for local control and acceptable anal function in a series of 35 patients (median follow-up: $23 \mathrm{mo}$ ), these patients had T3-staged ultra-low rectal cancer and were treated by preoperative CCRT with consecutive ISR[7].

In the past 4 years, 10 patients with ultra-low rectal cancer were managed in this study by multimodality treatment and no distant metastasis was disclosed during the follow-up period. Preoperative CCRT was applied to six T3-4 staged patients, and this treatment enhanced tumor shrinkage more than $25 \%$ in five patients; it is inspiring that no residual cancer was identified in 2 patients among these 5 patients. After a median follow-up of $30 \mathrm{mo}$, there was no local recurrence or distant metastasis developed.

Cluster defecation associated with tenesmus, urgency, and incontinence are not uncommon in straight coloanal anastomosis. The colonic pouch could convert the functional deficiencies associated with the loss of rectal capacity and reduced compliance resulting from straight coloanal anastomosis. Lazorthes et al. [16] and Parc et al. [17] conducted a colonic $\mathrm{J}$ pouch anastomosing at the dentate line, and they assumed superior functional outcome compared to straight anastomosis [16,17]. A number of clinical series have evidence to support that utilizing a colonic pouch anal anastomosis could enhance the functional results and postoperative quality of life of patients after rectal cancer resection [16-19, 21, 22]. The functional advantages of a colonic pouch anal anastomosis have been achieved within a shorter period than straight coloanal anastomosis after surgery. This superiority in $\mathrm{J}$ pouch groups remains evident when compared with the straight coloanal anastomosis 2 years after the surgery as reported by Joo et al. [21] and Sailer et al. [22].

In conclusion, ISR could achieve acceptable local control, cancer-related survival and avoid permanent stoma in patients with early staged ultra-low rectal cancer. Preoperative CCRT facilitates intersphincteric curative resection in patients with T3-4 staged ultra-low rectal cancer. Longer follow-up of these patients should be recommended, 
especially for those receiving preoperative chemoradiotherapy.

This study analyzes the safety and feasibility of laparoscopic surgery for rectal cancer including the shortterm results. Numerous studies have demonstrated that laparoscopic techniques have many advantages in colorectal surgery compared with open surgery. 13-15 However, it is still controversial to perform laparoscopic surgery for rectal cancer because of the difficulty in understanding the accurate anatomy of the small pelvic cavity, in dissecting the TME or TSME plane, and in transecting the lower rectum safely. This study demonstrated that laparoscopic surgery for rectal cancer was safe and feasible, and the 3-year disease-free survival rate was acceptable. Laparoscopic surgery did not jeopardize the perioperative outcomes and the short-term results compared with the results reported in the literature. A series reported by Lacy and colleagues showed that the laparoscopic approach had survival advantages over the conventional approach, 8 but they excluded the rectal cancer. This study analyzed only rectal cancer. Conversion from laparoscopic surgery to open surgery occurred because of technical difficulties, the presence of associated conditions such as obesity and unexpected adhesion, or findings of locally advanced disease. Conversion to open surgery should not be hesitated for the patients' safety. In our series, conversion rate was very low. The reported morbidity rates for laparoscopic colorectal surgery vary between 6 and 39 percent. 16-26 The postoperative morbidity in this study was mainly due to anastomotic leakage and wound infection, which was the same as reported in open surgery. The mortality rate within 30 days after surgery, which is one of the most important factors to analyze the safety of laparoscopic surgery, was not experienced in this study.
Low conversion rate and the acceptable morbidity and mortality rate shows that laparoscopic surgery itself was performed safely and feasibly. The postoperative hospital stay was not short in this study especially in cases with complications. Owing to the specifics of the health insurance system in our country, the duration of postoperative hospital stay cannot be compared with the data in the international literature. Adequate tumor resection and lymphadenectomy are major problems of cancer surgery. The number of lymph nodes harvested in this study was comparable with the studies so far published on laparoscopic resection. 26-30. The 3-year disease free survival rate was $100 \%$ in stage $0 /$ I patients, $89.1 \%$ in stage II and $84.6 \%$ in stage III. This result was acceptable and extremely favorable compared with that reported in the literature. [31-34] This study concentrated on the lesions at the rectum because rectum was one of the commonest sites of colorectal cancer which we encountered and we had many experiences in open surgery. Laparoscopic surgery has been reported to have a magnified view and get better visual fields than open surgery. However, in cases of lower rectal cancer it is sometimes difficult to grasp the correct dissecting plane and dissect the rectum in the pelvic cavity because of obesity, narrow pelvic space, and the tumor extension. This study clarified that the morbidity rate was acceptable, the mortality was not experienced, and short-term outcome was quite acceptable compared with open surgery. This study analyzed only small number of laparoscopic surgery retrospectively. Large number of cases with rectal cancer operated laparoscopically should be accumulated by multicenter study, and safety and feasibility of laparoscopic surgery for lower rectal cancer should be made sure. Based on the results of multi-center randomized control study is essential.

\section{REFERENCES:}

1. Zhao GP, Zhou ZG, Lei WZ, Yu YY, Wang C, Wang Z, et al. Pathological study of distal mesorectal cancer spread to determine a proper distal resection margin. World J Gastroenterol. 2005 Jan;11(3):319322. [PubMed]

2. Rullier E, Zerbib F, Laurent C, Bonnel C, Caudry M, Saric J, et al. Intersphincteric resection with excision of internal anal sphincter for conservative treatment of very low rectal cancer. Dis Colon Rectum. 1999 Sep;42(9):1168-1175 [PubMed]

3. Kohler A, Athanasiadis S, Ommer A, Psarakis E. Long-term results of low anterior resection with intersphincteric anastomosis in carcinoma of the lower onethird of the rectum: analysis of 31 patients.
Dis Colon Rectum. 2000 Jun;43(6):843850. ]PubMed]

4. Schiessel R, Karner-Hanusch J, Herbst F, Teleky B, Wunderlich M. Intersphincteric resection for low rectal tumours. Br J Surg. 1994 Sep;81(9):13761378. [PubMed]

5. Rullier E, Laurent C, Bretagnol F, Rullier A, Vendrely V, Zerbib F. Sphinctersaving resection for all rectal carcinomas: the end of the 2-cm distal rule. Ann Surg. 2005 Mar;241(3):465-469. [PubMed] [CrossRef]

6. Vorobiev GI, Odaryuk TS, Tsarkov PV, Talalakin AI, Rybakov EG. Resection of the rectum and total excision of the internal anal sphincter with smooth muscle plasty and colonic pouch for treatment of ultralow rectal carcinoma. Br J Surg. 2004 Nov;91(11):1506-1512. [CrossRef] [PubMed]

7. Saito N, Ono M, Sugito M, Ito M, Morihiro M, Kosugi C, et al. Early results of intersphincteric resection for patients with very low rectal cancer: an active approach to avoid a permanent colostomy. Dis Colon Rectum. 2004 Apr;47(4):459466. [PubMed]

8. Tiret E, Poupardin B, McNamara D, Dehni N, Parc R. Ultralow anterior resection with intersphincteric dissectionwhat is the limit of safe sphincter preservation? Colorectal Dis. 2003 Sep;5(5):454-457. [PubMed] [CrossRef]

9. Prete F, Sebastiani R, Sammarco DF, Prete FP. Role of inter-sphincter resection 
among the surgical options for cancer of the distal rectum. Chir Ital. 2001 NovDec;53(6):765-772. (in Italian) [PubMed]

10. Rullier E, Goffre B, Bonnel C, Zerbib F, Caudry M, Saric J. Preoperative radiochemotherapy and sphincter-saving resection for T3 carcinomas of the lower third of the rectum. Ann Surg. 2001 Nov;234(5):633-640. [PubMed]

11. Rullier E, Laurent C, Zerbib F, Belleannee G, Caudry M, Saric J. Conservative treatment of adenocarcinomas of the anorectal junction by preoperative radiotherapy and intersphincteral resection. Ann Chir. 2000 Sep;125(7):618-24. (Article in French) [PubMed] [CrossRef]

12. Parks AG, Percy JP. Rectal carcinoma: restorative resection using a sutured colo-anal anastomosis. Int Surg. 1983 Jan-Mar;68(1):7-11. [PubMed]

13. Luppi G, Santantonio M, Bertolini F, Fiorica F, Zanelli F, Gavioli M, et al. Preoperative concomitant radiotherapy and chemotherapy in ultrasound-staged T3 and T4 rectal cancer. Tumori. 2003 MarApr;89(2):152-156. [PubMed]

14. Habr-Gama A, Perez RO, Kiss DR, Rawet V, Scanavini A, Santinho PM, et al. Preoperative chemoradiation therapy for low rectal cancer. Impact on downstaging and sphincter-saving operations. Hepatogastroenterology. 2004 NovDec;51(60):1703-1707. [PubMed]

15. Gerard JP, Chapet O, Nemoz C, Hartweig J, Romestaing P, Coquard R, et al. Improved sphincter preservation in low rectal cancer with high-dose preoperative radiotherapy: the lyon R96-02 randomized trial. J Clin Oncol. 2004 Jun 15;22(12): 2404-2409. [PubMed] [CrossRef]

16. Lazorthes F, Fages P, Chiotasso P, Lemozy J, Bloom E. Resection of the rectum with construction of a colonic reservoir and colo-anal anastomosis for carcinoma of the rectum. Br J Surg. 1986 Feb;73(2):136-138. [PubMed]

17. Parc R, Tiret E, Frileux $P$, Moszkowski E, Loygue J. Resection and colo-anal anastomosis with colonic reservoir for rectal carcinoma. Br J Surg. 1986 Feb;73(2):139-141. [PubMed]

18. Bretagnol F, Rullier E, Laurent C, Zerbib F, Gontier R, Saric J. Comparison of functional results and quality of life between intersphincteric resection and conventional coloanal anastomosis for low rectal cancer. Dis Colon Rectum. 2004 Jun;47(6):832-838. Epub 2004 Apr 19.
[PubMed]

19. Amin AI, Hallbook O, Lee AJ, Sexton R, Moran BJ, Heald RJ. A 5-cm colonic $\mathrm{J}$ pouch colo-anal reconstruction following anterior resection for low rectal cancer results in acceptable evacuation and continence in the long term. Colorectal Dis. 2003 Jan;5(1):33-37. [PubMed] [CrossRef]

20. Hida J, Yasutomi M, Fujimoto K, Okuno K, Ieda S, Machidera N, et al. Functional outcome after low anterior resection with low anastomosis for rectal cancer using the colonic J-pouch. Prospective randomized study for determination of optimum pouch size. Dis Colon Rectum. 1996 Sep;39(9):986-991. [PubMed]

21. Joo JS, Latulippe JF, Alabaz O, Weiss EG, Nogueras JJ, Wexner SD. Longterm functional evaluation of straight coloanal anastomosis and colonic J-pouch: is the functional superiority of colonic Jpouch sustained? Dis Colon Rectum. 1998 Jun;41(6):740-746. [PubMed]

22. Sailer M, Fuchs KH, Fein M, Thiede A. Randomized clinical trial comparing quality of life after straight and pouch coloanal reconstruction. Br J Surg. 2002 Sep;89(9):1108-1117. [PubMed]

\section{Corresponding author:}

\section{Anton Tonev}

1st Clinic of Surgery, University Hospital "St. Marina",

1, Hristo Smirnensky str., Varna, 9000, Bulgaria

E-mail: teraton@abv.bg 